Journal of Machine Engineering, 2020, Vol. 20, No. 1, 107-116

ISSN 1895-7595 (Print) ISSN 2391-8071 (Online)

Received: 22 December 2019 / Accepted: 08 February 2020 / Published online: 05 March 2020

robotized additive manufacturing, long fibers filled polymers, fused filament fabrication

Jean-Yves HASCOËT ${ }^{1 *}$

Olivier AUTHELIN ${ }^{1}$

Matthieu RAUCH ${ }^{1}$

\title{
A GENERIC METHOD TO REALIZE LONG FIBERS FILLED AND LARGE POLYMER PARTS IN ADDITIVE MANUFACTURING
}

\begin{abstract}
A general manufacturing methodology for long fibers filled and large polymer (LFFLP) parts will be proposed, which constitutes the major scientific contribution of the document. The input, output, control and analysis data at each step of the methodology will be specified. Experiments realized in the laboratory of Ecole Centrale de Nantes will demonstrate the relevance and effectiveness of this method applied to a 6-axis robot and the FFF process by showcasing two light and resistant lattice structures. The latter also highlight the capacity of 6-axis robots for orienting the deposition head in order to generate complex trajectories. Finally, perspectives and future research about this subject will be discussed such as the need to develop in-depth analyses of the manufacturing methodology. The possibility of using continuous fibres composites as material feedstock for robotized large dimensions FFF will also be covered.
\end{abstract}

\section{INTRODUCTION}

Additive manufacturing is a manufacturing process which consists in constructing parts by successive addition of material layers. The latter makes it possible to obtain parts of various nature, going from metals to composite materials as well as parts with gradients of materials [1]. Today, the knowledge related to additive manufacturing is vast: generation of toolpaths [2], design for additive manufacturing (DFAM) [3], hybrid manufacturing processes [4] and many others.

Fused Filament Fabrication (FFF), also known as Fused Deposition Modeling (FDM), is a thermoplastic polymer-based additive manufacturing process [5]. Initially limited to prototyping, this technology now allows realizing parts of structure. However, the current 3-axis cartesian FFF process has limitations on parts with complex geometries including overhangs, draft and undercut angles [6]. Indeed, freedom of structural creation is limited by the constraint on the deposition tool axis which must remain vertical. Pieces made in this

\footnotetext{
${ }^{1}$ Institut de recherche en Génie Civil et Mécanique, Ecole Centrale de Nantes, France

*E-mail: jean-yves.hascoet@ec-nantes.fr https://doi.org/10.36897/jme/118217
} 
manner are called $2.5 \mathrm{D}$ printing because the layers remain horizontal and stacked along a vertical axis. In addition, the mechanical and geometric properties of parts with curved or inclined surfaces are altered. The so-called "staircase effect", caused by the superposition of horizontal planes, has the effect of increasing the volume difference with respect to the reference digital model and degrading the surface quality of the fabricated structures [7]. When making hull-type inclined surfaces, the contact surface between the layers is reduced. This reduces the conductive exchanges and thus weakens the adhesion force between the planes.

Nowadays, a big step is being made by using 6-axis robots instead of more usual cartesian machines. Otherwise known as anthropomorphic robots, these have been used for many years for applications requiring accurate trajectory tracking in the context of milling, painting deposition or welding [8-10]. At the Rapid Manufacturing Platform (Laboratory GeM, Ecole Centrale de Nantes), the Wire Arc Additive Manufacturing process is used to make large and complex metal alloys parts. Several projects demonstrating the capabilities of robotic additive manufacturing have been conducted: A hollow propeller blade, intended for the marine industry, has been realized. The latter has a height of $1.5 \mathrm{~m}$, a span of $1.5 \mathrm{~m}$ and weighs $800 \mathrm{~kg}$. A fuselage element for the aerospace industry consisting of a double curved thin plate, on which primary and secondary stiffeners were made, was produced in collaboration with Stelia company [11]. This trajectory tracking and the benefits that come with it are the reasons why 6-axis robots bring added value to the FFF process: the possibility of following a path in space while keeping the control of the final effector (orientation, position) allows material deposition planes to be oriented arbitrarily. The homogeneity of the material deposition is guaranteed by the robot's ability to maintain a constant speed, irrespective of its position and orientation in space. Moreover, the working volumes of the robots allow the realization of large and complex, which can be suitable for aeronautical or naval applications.

In the second section, a state of the art concerning the robotized FFF materials and fields of application will be presented. A summary exposing the conclusions of the state of the art as well as the positioning of the article will highlight the lack of scientific knowledge and the need for a manufacturing methodology for the LFFLP parts manufacturing. The choice to use a 6-axis robot to produce the latter was made. In the third section, a generic manufacturing methodology adapted to LFFLP parts will be proposed. This methodology was proposed based on literature review as well as on feedback from experimental studies carried out in the laboratory. Application cases, presented in the fourth section, will underline the relevance of this method.

\section{STATE OF THE ART}

\subsection{ROBOTIZED FFF MATERIALS}

A wide variety of polymer materials are used with the robotic FFF process, from less expensive to more technical. The vast majority of these belong to the category of thermoplastics, which can be melted and solidified very quickly. The most used materials 
are Acrylonitrile Butadiene Styrene (ABS), Polypropylene (PP) and Polylactic Acid (PLA). Composite materials are also used because of the mechanical properties they give to the parts. The nature (glass, carbon, aramid, Kevlar) and the length (short, long or continuous fibres) of the reinforcement are chosen according to the type of application to which the piece will be confronted [12-14]. The possibility of using long or continuous fibres opens the way towards the fabrication of large composite parts, as this kind of reinforcement allows to considerably increase manufacturing volumes while decreasing the overall mass without deterioration of mechanical properties [15].

\subsection{ROBOTIZED FFF FIELDS OF APPLICATIONS}

The fields of application of the robotized FFF are varied, ranging from the construction industry to design and architecture. Many projects are in progress or have been completed in research laboratories and private companies. Within the AI Build Company (London, England) are Kuka robots building large polymers lattice structures. The company has developed its own software, deposition head and printing cell. The start-up Drawn (Lyon, France) produces on demand, custom made furnishing pieces such as chairs, benches or pieces of decorations by using an $\mathrm{ABB}$ robot. Some projects are inspired by bio mimicry to create audacious structures. Researchers from the Tongji University, China, used a 6-axis Kuka robot to overcome gravity and overhang limits by creating a structure inspired by spider web[16]. The principle is as follows: around the freeform main curve are attached three wavy auxiliary curves, distant from an angle of 120 degrees. This allows the structure to have a higher bending resistance. Branch technology (Chattanooga, USA) is using a 6-axis Kuka robot, placed on a rail to increase the work envelop, to manufacture a large dimensions lattice structure made of short carbon fibres - ABS for the construction market.

\subsection{LONG FIBERS FILLED AND LARGE POLYMER PARTS APPLICATIONS}

In Switzerland, a research laboratory is working on high-performance FFF composite materials coupled with innovative structures. ETH Zurich developed a pultrusion - extrusion robotized additive manufacturing process (called Continuous Lattice Fabrication) in which long carbon fibres, surrounded by polyamide 12, are deposited in order to build lightweight and compression resistant structures, like the one in Fig. 1 [17].

State Key Laboratory for Manufacturing Systems Engineering (Xi'an Jiaotong University, China) has created robotic corrugated structures made with reinforcements based on continuous carbon fibers and a PLA matrix[18]. The fracture modes during compression loading were analyzed and demonstrated the importance of density and fiber content in order to improve compressive strength. Subsequently, work was carried out to study the effect of process pressure and temperature on the interface and performances of printed composites. It has been shown that by increasing the fiber content in the composite, the resistance and the modulus of flexion are improved [19]. 


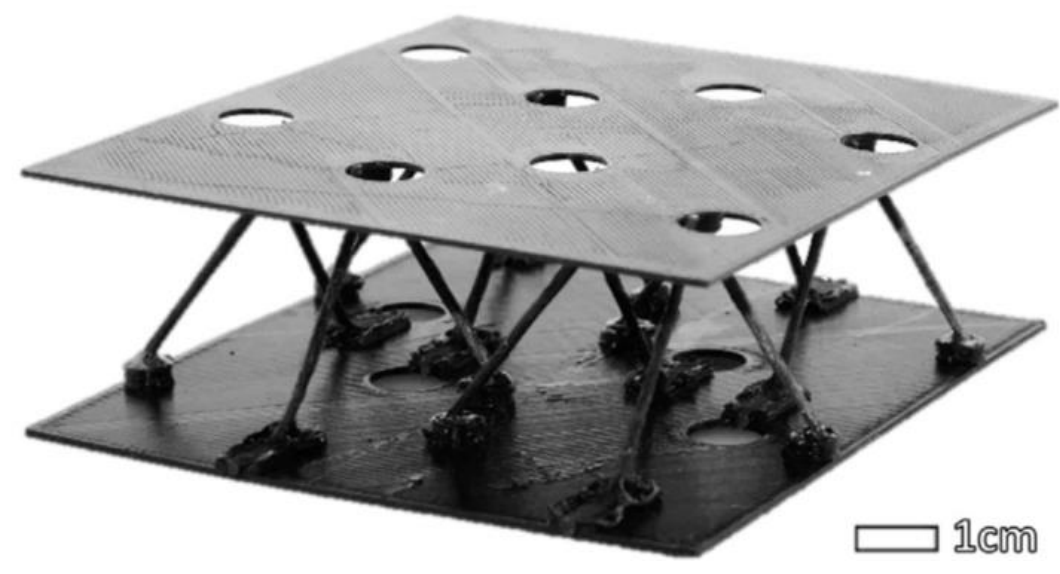

Fig. 1. Ultra-light FFF structure, CLF process [17]

\subsection{SYNTHESIS}

To the authors knowledge, there are no applications in the literature of LFFLP parts made using the robotic FFF process for the aerospace and shipbuilding industries, which can be explained by two reasons. First, the research knowledge on long and continuous fiber composite materials as part of this process is not sufficiently advanced. Methods relating to DFAM, operating parameters definition and toolpaths generation for long fibers filled and large polymer (LFFLP) parts must be developed. As these industries use composite materials at the cutting edge of technology, progress must be made in this field. Moreover, in order to solve the delamination problems inherent in additively manufactured polymer parts, continuous fibers filled polymer material can be deposited transversely to the deposition planes, thus reinforcing the part properties in this direction. Second, there is no clear and precise methodology to follow step by step the path leading to obtain a large and complex long fibers filled polymer FFF part. To fill that lack of knowledge, a methodology for manufacturing large and complex composite parts will be proposed in the following section. This method, based on literature review and experimental studies, is generic and may be applied regardless of the means or the manufacturing process. Within the laboratory, the choice to use a 6-axis robot in order to produce LFFLP parts was made. Applications, using a Staübli robot and FFF process, demonstrating the effectiveness of this method will then be presented. The latter also highlight robotic toolpaths generation capabilities.

\section{LARGE AND LONG FIBERS FILLED PART MANUFACTURING METHODOLOGY}

The LFFLP parts manufacturing methodology aims to be generic and global. It allows moving forward, step by step, from the digital model to the part manufacturing passing by toolpaths generation, in a digitally integrated manufacturing environment, and process parametrization while keeping a global vision of the approach and the objective to be achieved. 
The LFFLP parts manufacturing methodology takes into account the dimensional, kinematic and dynamic constraints generated by the choice of manufacturing mean. Part design, toolpaths as well as operating parameters are adapted to it. Indeed, the toolpaths followed by a 6-axis robot differ from those made by a Cartesian manufacturing means or a 5-axis machine. The manufacturing methodology also takes into account the manufacturing process and the constraints that this latter implies. For example, pultrusion process deposition head necessary to extrude material requires equipment and operating parameters different from those used with the FFF process. Finally, long or continuous fibers reinforced material generates constraints in terms of toolpaths generation, process parametrization as well as in terms of required deposition head equipment. A particular attention is paid to this effect.

The IDEF0 diagram, presented in Fig. 2, represents the different stages of the manufacturing methodology. The steps to be followed are represented by blue rectangles (A1 - DFAM, A2 - Process parametrization, A3 - Manufacturing, A4 - Control). The control data, which impose and set constraints on the different stages of the methodology, is above (material capacity, mean capacity, process). The analysis methods on which the choices made during the different stages of the methodology are based are located below each of the steps. Red framed analysis methods (DFAM analysis, toolpath definition and deposition head design for LFFLP parts) represent the scientific locks that need to be removed in future research. The latter will be specially developed to produce large and long fiber filled polymer parts.

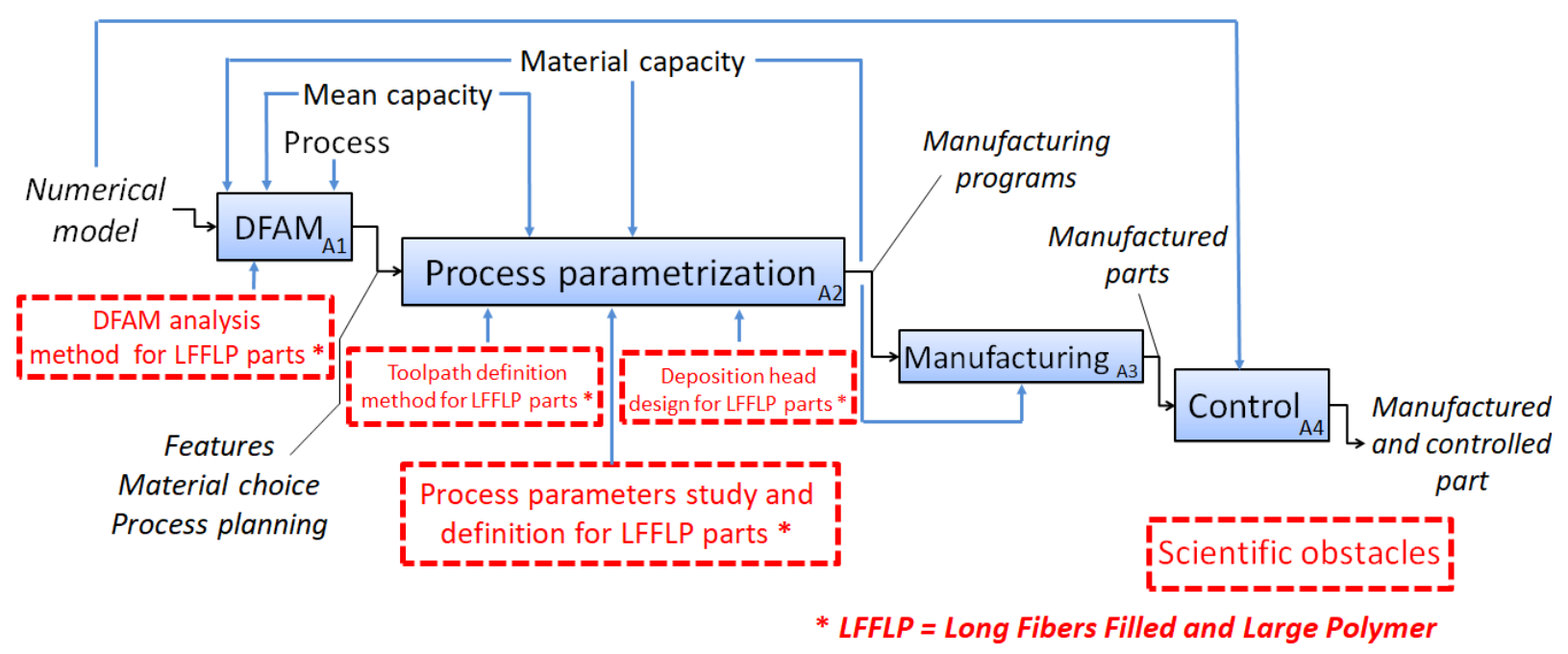

Fig. 2. Large long fibers filled parts methodology (IDEF0)

In the first step of the manufacturing methodology, digital model is divided into elementary entities, called features thanks to LFFLP parts specific DFAM analysis (A1). The latter depends on parameters specific to the manufacturing mean, materials and process used. LFFLP part FDAM method determines digital model elementary manufacturing volumes in which are informed the manufacturing strategy (layers slicing, deposition head frame orientation, reinforcing fibers placement) and materials used. Features manufacturing cycles can be carried out one after the other or in parallel, depending on the geometries to be produced. This information is contained in the process planning. 
The process parametrization step, which corresponds to the IDEF1 diagram detailed in Fig. 3, consists of three sub-steps. First, the process parameters are chosen (temperatures, feed rates, spacing, layer thickness) based on LFFLP parts specific process parameters study and definition method (A21). Parameters are chosen taking into account the manufacturing mean and the materials used. These are based on literature, feedback and simulation analysis. Then, toolpaths are defined using the LFFLP specific toolpath definition method (A22). This method allows determining the optimal patterns and parameters for filling and contouring, taking into account the materials constraints and the manufacturing mean used. The steps for choosing the manufacturing parameters and toolpath definition both depend on the design of the deposition head adapted to LFFLP. The latter must have a shape which optimizes the toolpaths generation while taking into account the composite material nature and process parameters to reach.

Once the parameters and trajectories have been chosen, a simulation step (A23) is carried out in order to verify that manufacturing cycles are operating correctly. Kinematic simulation lets you know if the robot is facing singularities, collisions or axis limits when tracking the deposit paths. Mechanical simulation allows anticipating the presence of residual stresses within the part while thermal simulation indicates the presence of distortions during structure cooling. These simulations are based on multi-physical and kinematic models. The data coming out of the simulations allows analyzing and correcting the process parameters and generated toolpath.

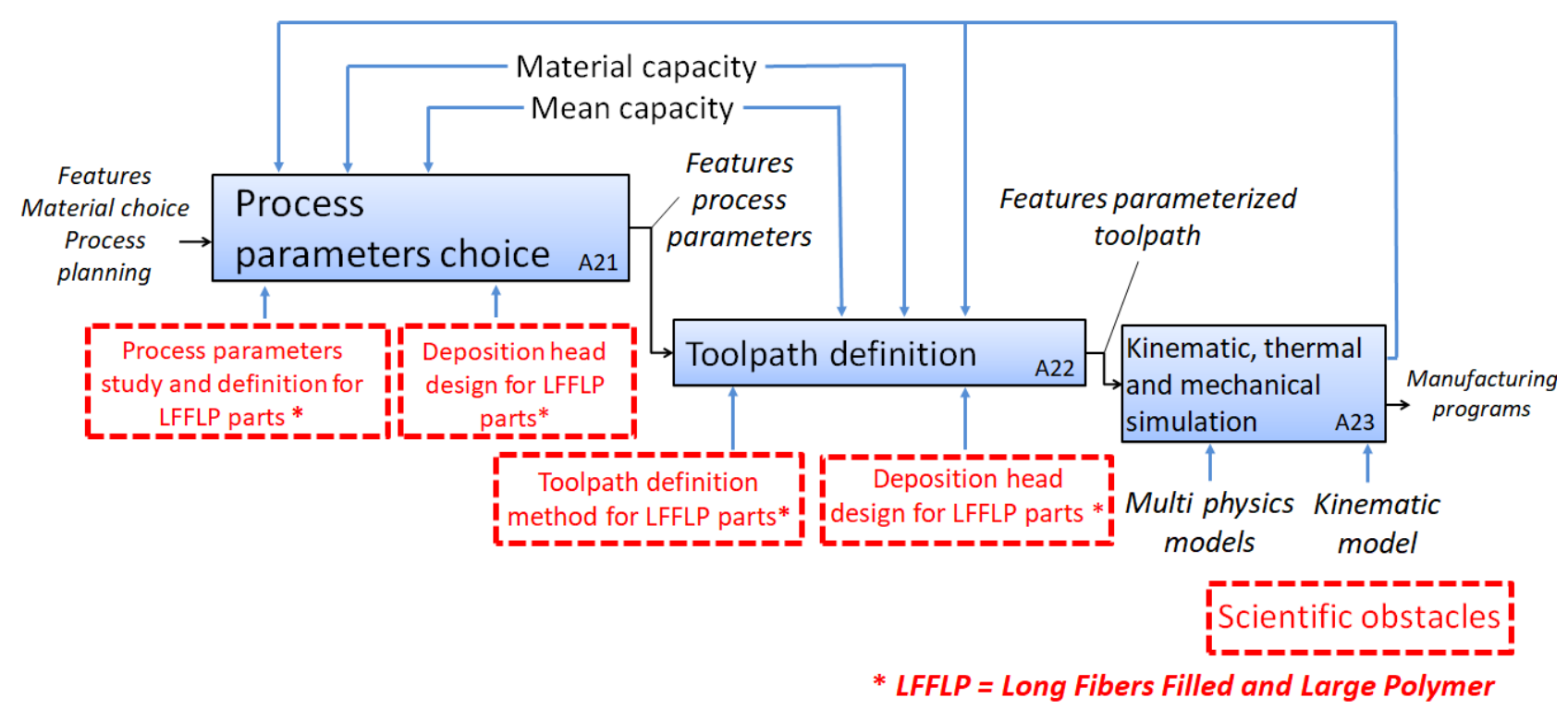

Fig. 3. Process parametrization methodology (IDEF1)

At this stage, the manufacturing programs are generated and the manufacturing cycle can be completed (A3). A control step (A4) is conducted in order to verify the part accuracy by comparing the latter with the digital model. A $3 \mathrm{D}$ reconstruction of the part obtained can be done as part of non-destructive tests. Once the control step is finished, the manufacturing cycle is completed. 


\section{APPLICATIONS}

The manufacturing methodology presented in the previous section is general and can be applied whatever the means or the process. The following application cases, using a 6-axis robot as a manufacturing mean and FFF process, were carried out following the manufacturing methodology presented in the previous section in order to prove its effectiveness and relevance on practical applications. These also highlight the ability of a 6-axis robot to generate complexes as well as light and resistant structures. However, the material used is not reinforced with long fibers, scientific locks preventing us for the moment from using them wisely. The experiments have been realized within the Rapid Manufacturing Platform (RMP, GeM laboratory, Ecole Centrale de Nantes). The experimental set-up illustrated in Fig. 4 consists of the following elements: a base down Stäubli robot at the end of which has been attached a deposition head made in the laboratory.

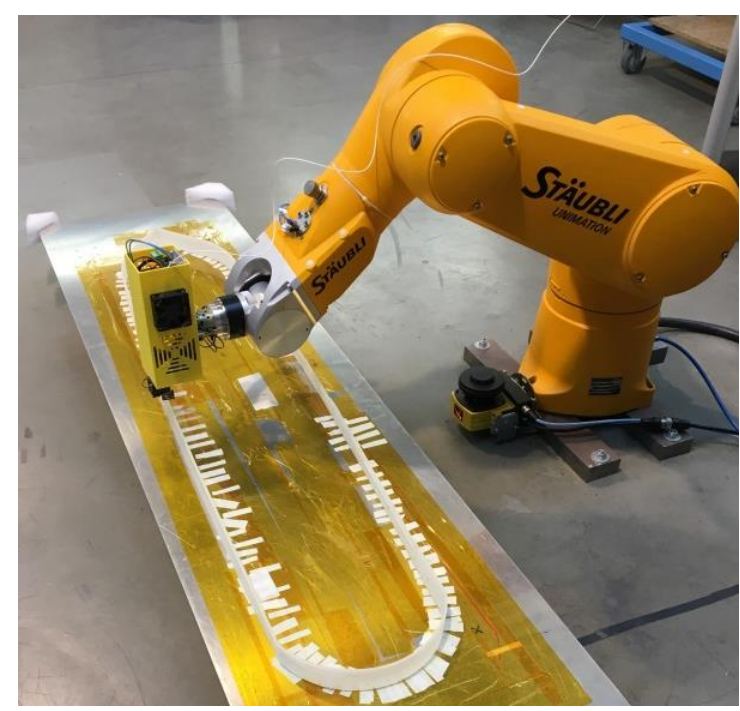

Fig. 4. Experimental setup

The structures, shown in Fig. 5, have the following dimensions: the bottom square base has $270 \mathrm{~mm}$ length side, the upper square base has a $70 \mathrm{~mm}$ length side and the total height is $120 \mathrm{~mm}$. The structure on the right, illustrated in Fig 5, is the same than the one on the left with diagonal beams added. These will be presented simultaneously as they have the same manufacturing parameters.

Below will be presented the steps in chronological order of the manufacturing methodology presented in the previous section.

A1, DFAM:

The structures consist of 6 superimposed material layers that follow a continuous, single-track tool path, so that the decomposition into elementary volumes is not necessary. The structures were made of Polylactic Acid (PLA) stored on coils in the form of filaments with a diameter of $1.75 \mathrm{~mm}$. Reinforcements are not present in these experimental tests. As the toolpath were a continuous single-track toolpath, no spacing had to be defined. 


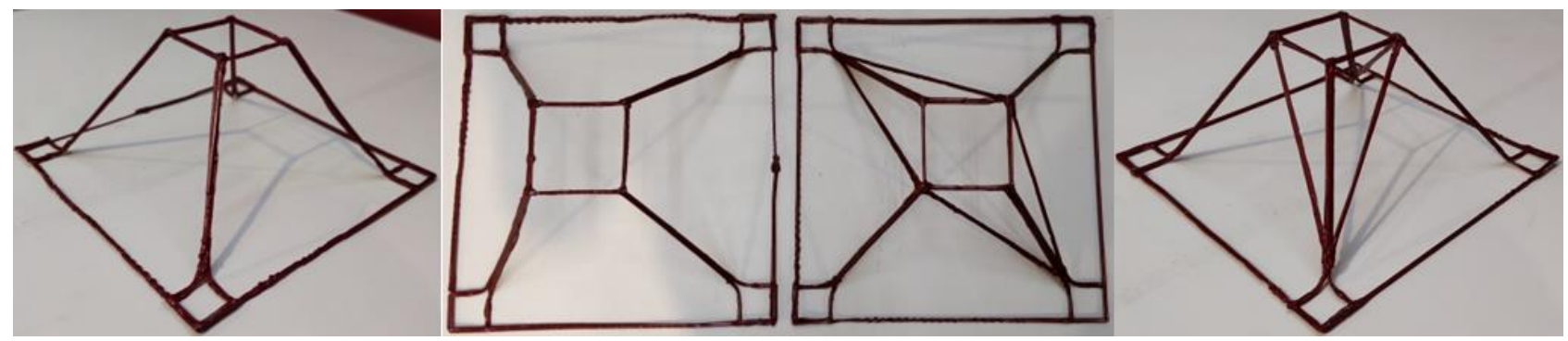

Fig. 5. Experimental results

\section{A2, process parametrization:}

A21, process parameters: Nozzle temperature $\left(190^{\circ} \mathrm{C}\right)$, layer thickness $(0.4 \mathrm{~mm})$, feed rate (deposition: $0.3 \mathrm{~m} / \mathrm{min}$ ) were defined based on the results of experimental studies. The temperature, during the experiments, was $23^{\circ} \mathrm{C}$ and the atmosphere was not controlled.

A22, toolpath definition:

The tool axis (deposition head frame $Z$ axis) is constrained to remain perpendicular to the toolpath, as shown in Fig. 6: This orientation maximizes the force applied by the deposition head on the previous layers and therefore the bonding between them [20]. The orientation vector (deposition head frame $X$ axis), which corresponds to the tool direction of advance, remains free in order to reduce the orientation constraints of the deposition head. The orientation vector is to be parameterized in the case of non-cylindrical injection nozzle outlet section, which is not the case. Since the toolpath is of continuous single track type, the following parameters have not been defined: off-material movements, contouring passes, filling passes.

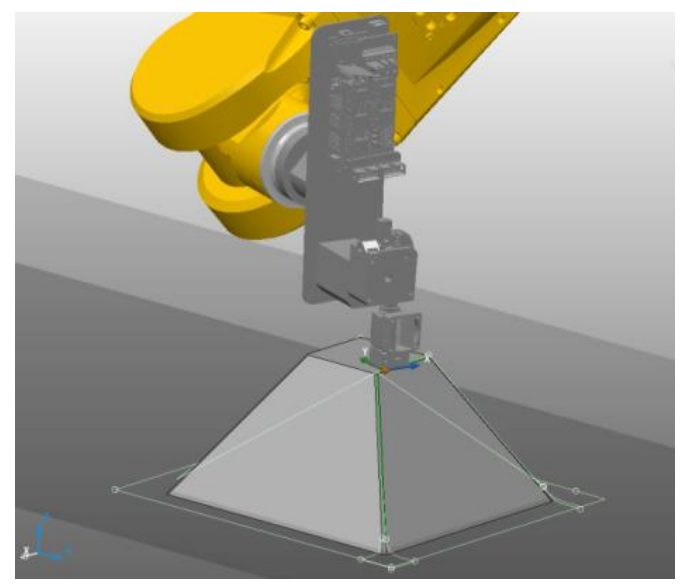

Fig. 6. Toolpath definition (A22)

\section{A23, simulation stage:}

Manufacturing cycle kinematic analyis did not reveal any collisions between the robot and the support. The axis limits of the robot have not been reached. On the other hand, the presence of wrist singularity (collinearity of axes 4 and 5) forced the robot to change its posture during the manufacturing cycle, especially at the top and bottom of the inclined edges. Mechanical and thermal simulations were not performed during the trials. 


\section{A3, manufacturing:}

Manufacturing cycle went smoothly except during singularity phases. The structures manufacturing, illustrated in Fig. 7, showed that the results obtained during the kinematic analysis phase were correct, which underlines the importance of this step.

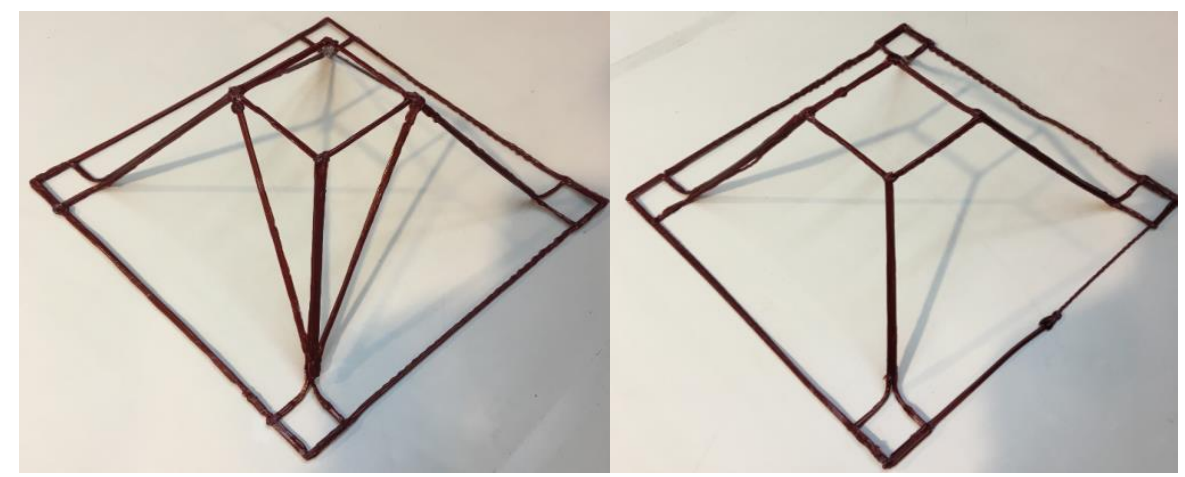

Fig. 7. Manufacturing (A3)

\section{A4, control:}

The dimensions post manufacturing control of the structure was not carried out as part of these experimental tests.

These experimental tests highlight the relevance and importance of using a global manufacturing methodology to break down, step by step, the sequence of actions required to manufacture a LFFLP parts made by the robotized FFF process. The trajectories carried out as part of these experimental tests also illustrate the advantages of using a 6-axis robot in the context of the FFF process: deposition planes are inclined and oriented in order to obtain complex geometries, in this case light and resistant lattice structures (structure without cross beams has a weight-to-mass ratio of 156 while the structure with cross beams has a weightto-mass ratio of 190). This kind of realization would not have been possible by using cartesian deposition means because the tool axis is constrained to remain vertical.

\section{CONCLUSION}

This paper highlighted the weaknesses of the FFF process using cartesian machines and the benefits related to the use of 6-axis robots to create structures with complex geometries. The freedom of orientation and inclination of the deposition planes, through the control of the deposition head frame, maximizes the mechanical and geometrical properties of the parts produced while increasing the freedom of structural creation. Thus light and resistant lattice structures, such as those highlighted in the applications section, or parts with external or internal curved or cantilevered surfaces can be produced without lowering the overall quality of manufacture.

A general methodology, presenting step by step the actions to carry out to realize LFFLP parts was presented. The latter makes it possible to take into account all of the manufacturing cycle aspects, from the toolpaths generation to the robotic, mechanical and thermal simulation 
passing through the parametrization of the process. Application cases, using a 6-axis robot and the FFF process, highlighted the manufacturing methodology capabilities and the analysis methods to be implemented. Future research is to be carried out with the aim of developing analytical methods concerning DFAM, process parameters and deposition head design for LFFLP parts. This will be the subject of future work. Then, parts using continuous carbon fibers and high performance polymers could be developed, which is of great interest in the context of aeronautical and marine applications.

\section{REFERENCES}

[1] MULLER P., HASCOET J.Y., 2014, Toolpaths for additive manufacturing of functionally graded materials (FGM) parts, Rapid Prototyp. J., 20/6, 511-522.

[2] JIN Y., HE Y., FU J., GAN W., LIN Z., 2014, Optimization of tool-pa th generation for material extrusion-based additive manufacturing technology, Addit. Manuf., 1-4, 32-47.

[3] TAYLOR P., PONCHE R.J., HASCOET Y., KERBRAT O., MOGNO1 P., 2012, Virtual and Physical Prototyping A new global approach to design for additive manufacturing, Virtual Phys. Prototyp, 7/2, 93-105.

[4] KERBRAT O., MOGNOL P., HASCOE J., 2011, Computers in Industry a new DFM approach to combine machining and additive manufacturing, Comput. Ind., 62, 684-692.

[5] TURNER B.N., STRONG R., GOLD S.A., 2014, A review of melt extrusion additive manufacturing processes: I. Process design and modeling, Rapid Prototyp. J., 20/3, 192-204.

[6] ZHAO H., HE Y., FU J., QIU J., 2018, Inclined layer printing for fused deposition modeling without assisted supporting structure, Robot. Comput. Integr. Manuf., 51, 1-13.

[7] AHN D., KWEON J., KWON S., SONG J., LEE S., 2009, Representation of surface roughness in fused deposition modeling, J. Mater. Process. Technol., 209, 5593-5600.

[8] MUZAN I.W., FAISAL T., IWAN M., 2012, Implementation of Industrial Robot for Painting Applications, Procedia Eng., 41, 1329-1335.

[9] HASCOËT J., QUERARD V., RAUCH M., 2017, Interests of 5 axis toolpaths generation for wire arc additive manufacturing of aluminium alloys, Journal of Machine Engineering, 17/3,51-65.

[10] HAGE H., BIDAUD P., JARDIN N., 2012, Simulation of a Stäubli TX90 Robot during Milling Using SimMechanics, Appl. Mech. Mater., 162, 403-412.

[11] QUERARD V., HASCOËT J.Y, RAUCH M., 2019, Réalisation de pièces aéronautiques de grandes dimensions par fabrication additive WAAM, Centrale Nantes, France.

[12] KABIR S.M.F., MATHUR K., SEYAM M., 2020, A critical review on 3D printed continuous fiber-reinforced composites: History, mechanism, materials and properties, Compos. Struct., 232, 111476.

[13] MORI K., MAENO T., NAKAGAWA Y., 2014, Dieless forming of carbon fibre reinforced plastic parts using 3D printer, Procedia Eng., 81, 1595-1600.

[14] NAKAGAWA Y., MORI K., MAENO T., 2017, 3D printing of carbon fibre-reinforced plastic parts, Int. J. Adv. Manuf. Technol., 91, 2811-2817.

[15] LOVE L.J. et al., 2014, The importance of carbon fiber to polymer additive manufacturing, J. Mater. Res., 29/17, 1893-1898.

[16] YUAN P.F., MENG H., YU L., ZHANG L., 2016, Robotic Multi-dimensional Printing Based on Structural Performance, Robot. Fabr. Archit. Art Des., 92-105.

[17] EICHENHOFER M., WONG J.C.H., ERMANNI P., 2017, Continuous lattice fabrication of ultra-lightweight composite structures, Addit. Manuf., 18, 48-57.

[18] HOU Z., TIAN X., ZHANG J., LI D., 2018, 3D printed continuous fibre reinforced composite corrugated structure, Compos. Struct., 184, 1005-1010.

[19] TIAN X., HOU Z., LI D., LU B., 2016, 3D printing of continuous fiber reinforced composites with a robotic system for potential space applications, i-SAIRAS.

[20] UNQ S.G., RIZVI M., BELLEHUMEUR T., GU P., 2008, Effect of processing conditions on the bonding, Rapid Prototyp. J., 14/2, 72-80. 